\title{
VEGF receptor promotes hypoxia-induced hematopoietic progenitor proliferation and differentiation
}

\section{Jonathan Florentin}

University of Pittsburgh

Scott P. O’Neil

University of Pittsburgh

Sathish Babu Vasamsetti

University of Pittsburgh

Lee L. Ohayon

University of Pittsburgh

Anagha Arunkumar

University of Pittsburgh

Samit Ghosh

University of Pittsburgh

Jennifer C. Boatz

University of Pittsburgh

Justin Sui

University of Pittsburgh

Corrine R. Kliment

University of Pittsburgh

Stephen Y. Chan

University of Pittsburgh

Partha Dutta ( $\nabla$ duttapa@pitt.edu )

University of Pittsburgh

\section{Research Article}

Keywords: obstructive sleep apnea (OSA), chronic obstructive pulmonary disease (COPD), VEGF, metabolic, cardiovascular, neoplastic, neurologic disease

Posted Date: October 27th, 2021

DOl: https://doi.org/10.21203/rs.3.rs-1000955/v1 
License: (c) (i) This work is licensed under a Creative Commons Attribution 4.0 International License. Read Full License 
VEGF receptor promotes hypoxia-induced hematopoietic progenitor proliferation and differentiation

Jonathan Florentin $^{1 *}$, Scott P. O’Neil ${ }^{*}$, Sathish Babu Vasamsetti ${ }^{1}$, Lee L. Ohayon ${ }^{1}$, Anagha Arunkumar $^{1}$, Samit Ghosh ${ }^{2}$, Jennifer C. Boatz ${ }^{2}$, Justin Sui ${ }^{2}$, Corrine R. Kliment ${ }^{2}$, Stephen Y. Chan ${ }^{1}$, Partha Dutta ${ }^{1,3}$

1 Pittsburgh Heart, Lung, Blood, and Vascular Medicine Institute, Division of Cardiology, Department of Medicine, University of Pittsburgh School of Medicine, University of Pittsburgh Medical Center, Pittsburgh, PA 15213

${ }^{2}$ Department of Medicine, Division of Pulmonary and Critical Care, University of Pittsburgh, Pittsburgh, PA 15260, USA

${ }^{3}$ Department of Immunology, University of Pittsburgh School of Medicine, Pittsburgh, PA 15213

${ }^{*}$ These authors contributed equally to this work.

Corresponding Author:

Dr. Partha Dutta, DVM, PhD

Assistant Professor of Medicine and Immunology

Vascular Medicine Institute

Division of Cardiology, Department of Medicine University of Pittsburgh Medical Center

BST 1720.1

200 Lothrop Street

Pittsburgh, PA 15213, USA

Tel: 412-383-7277

Email: duttapa@pitt.edu 


\begin{abstract}
Intermittent or chronic hypoxia is associated with morbidity and mortality in many diseases such as obstructive sleep apnea (OSA) and chronic obstructive pulmonary disease (COPD), respectively. We found exaggerated inflammation with heightened numbers of inflammatory leukocytes in these patients compared with age-matched controls. Chronically hypoxic mice exhibited similar trend and induced proliferation of hematopoietic stem and progenitor cells (HSPC), precursors of inflammatory leukocytes. Consistently, a transcriptomic analysis of human HSPC exposed to hypoxic conditions revealed elevated expression of genes involved in progenitor mobilization. Additionally, bone marrow cells in mice under hypoxic conditions expressed high amount of vascular endothelial growth factor (VEGF), VEGF receptor (VEGFr) and its target genes in hypoxic conditions. In silico and ChIP experiments demonstrated that HIF-1 $\alpha$ binds to the promoter region of $V E G F r$. These results indicate that VEGF signaling in HSPC is an important mediator of their proliferation and differentiation in hypoxia-induced inflammation and represents a potential therapeutic target to prevent aberrant inflammation in hypoxia-associated diseases.
\end{abstract}




\section{Introduction}

Diseases characterized by alveolar hypoxia, such as chronic obstructive pulmonary disease (COPD) and obstructive sleep apnea (OSA), are highly prevalent and contribute to significant morbidity and mortality around the world ${ }^{1-5}$. OSA is defined by intermittent nocturnal apneic or hypopneic events that result in impaired alveolar ventilation and subsequent hypoxemia ${ }^{6}$. COPD is a highly heterogenous condition characterized by airway inflammation and progressive obstruction due to noxious environmental exposures (e.g., cigarette smoke), which result in chronic subclinical and acute severe hypoxia ${ }^{1}$. There is a notable overlap in COPD and OSA comorbidities including metabolic, cardiovascular, neoplastic and neurologic disease, which a growing body of literature attributes to systemic inflammation ${ }^{1,7-9}$. To date, studies of alveolar hypoxia induced inflammation have focused primarily on the role of resident macrophages, and their interaction with alveolar endothelial cells mediating production of inflammatory cytokines ${ }^{10-13}$. Monocyte chemoattractant protein 1 (MCP-1), c-reactive protein, tumor necrosis factor alpha (TNF $\alpha$ ), IL-1, IL-6 and IL-8 are increased in hypoxic lung diseases and generally correlate with severity of disease ${ }^{12,14,15}$. In both OSA and COPD, correction of alveolar hypoxia, with nocturnal continuous positive airway pressure and supplemental oxygen, respectively, remain the mainstay therapies ${ }^{6,16-18}$. While there is strong evidence that local and systemic inflammation are key components of hypoxic diseases, a mechanistic role of hypoxia in exaggerated inflammation has yet to be defined.

Mounting evidence has demonstrated key inflammatory cells involved in the pathogenesis of various diseases including insulin resistance and atherosclerosis ${ }^{4,19-21}$. These cells have been proposed to originate from hematopoietic stem and progenitor cells (HSPC) in the bone marrow and spleen ${ }^{22-25}$. Patients with hypoxic lung disease exhibit elevated inflammation and leukocytosis. 
The leukocytosis observed in chronic inflammation likely represents production of inflammatory leukocytes by their progenitors. However, a direct link between hypoxia induced systemic inflammation and proliferation of HSPC has not been established. Furthermore, the mechanisms of HSPC proliferation and differentiation in hypoxia have not been studied.

Vascular endothelial growth factor (VEGF) has been identified as an important regulator of neovascularization by encouraging endothelial cell proliferation in various diseases such as agerelated macular degeneration and neoplastic diseases ${ }^{26-29}$. A number of recent studies have demonstrated elevated VEGF concentrations in peripheral blood of patients with hypoxic lung disease, which closely correlated with severity of disease ${ }^{30-35}$. However, the role of VEGF in HSC proliferation and inflammatory leukocyte production is not known.

Patients with OSA and COPD, characterized by alveolar hypoxia, displayed increased circulating myeloid cell numbers. We used two independent mouse models of hypoxia to understand the effect of hypoxia in HSPC proliferation: $\mathrm{C} 57 \mathrm{BL} / 6$ mice exposed to A) $10 \% \mathrm{O}_{2}$ for three weeks, an established mouse model of chronic hypoxia ${ }^{36-38}$ and B) cigarette smoke for six months, a COPD mouse model ${ }^{39,40}$. Mice under chronic hypoxic conditions and exposed to cigarette smoke had elevated numbers of neutrophils, monocytes and macrophages in the blood and lungs suggesting augmented hematopoiesis. In line with this, a whole genome RNA sequencing analysis of human HSPC cultured under hypoxic conditions showed increased expression of the genes involved in hematopoiesis. The number and proliferation of HSPC were increased when mice were exposed to hypoxia or cigarette smoke. Concomitantly, we measured increased expression of VEGFr in these activated progenitors indicating a possible role of VEGFr in hematopoiesis. Indeed, VEGFr 
blockade in vitro and in vivo diminished HSPC proliferation and their differentiation into myeloid cells, resulting in attenuated inflammation in hypoxic conditions. Mechanistically, we observed that HIF-1 $\alpha$ binds to the $V E G F r$ promoter and increases its expression in HSPC under hypoxic conditions. HIFla silencing reduced HSPC proliferation and their differentiation into inflammatory leukocytes.

As a whole, the present study describes a role of the VEGFr in the activation of hematopoietic progenitors and the production of inflammatory myeloid cells in hypoxia. Additionally, this work proposes the VEGFr as a possible therapeutic target to attenuate inflammatory burden in patients with hypoxia. 


\section{Results}

Patients and animals with hypoxia have elevated numbers of inflammatory leukocytes in the blood. Various diseases associated with intermittent or chronic hypoxia are characterized by exaggerated systemic inflammation ${ }^{5-7,20,21,41-43}$. Leukocytes, primarily myeloid cells, play a major role in inflammatory diseases including atherosclerosis and diabetes ${ }^{6,19}$. To ascertain systemic inflammation in patients with hypoxic lung diseases, we performed a retrospective chart review of patients with OSA characterized by intermittent hypoxia and COPD marked by chronic hypoxia, and age-matched control patients to enumerate inflammatory cells. The total numbers of neutrophils, lymphocytes, and monocytes were significantly increased in the patients with OSA and COPD compared to the healthy controls (Figure $1 \&$ Suppl. Figure 1A). The level of intermittent hypoxia has been shown to be linked with oxygen saturation or SpO2. Multiple studies evaluated $\mathrm{SpO}_{2}$ in patients with COPD and OSA. The average lung $\mathrm{SpO}_{2}$ during the daytime is $89 \%$ in COPD patients ${ }^{44,45}$. An OSA patient is diagnosed to have $\mathrm{SpO}_{2} \leq 90 \%$, and a number of desaturation events and patterns ( $\geq 3$ similar desaturation/saturation cycles, 15 min time scale) are $\geq 4$ and 12 , respectively ${ }^{46}$. Of note, the oxygen saturation levels of OSA and COPD patients included in this study were significantly reduced compared to healthy controls (Suppl. Figure 1B), indicating hypoxia in these patients. These results were confirmed in hypoxic mice which had a significantly lower oxygen saturation than the normoxic controls (Suppl. Figure 1C). Additionally, to understand if COPD elevates VEGFR expression in hematopoietic progenitors and increases lineage output, we employed C57BL/6 mice exposed to cigarette smoke for six months, an established mouse model of COPD ${ }^{40,47}$. These mice contained significantly heightened numbers of lymphocytes, such as B and T cells, in the bone marrow while myeloid cell content decreased (Suppl. Figure 1D). In contrast, splenic lymphocyte numbers were unaltered (Suppl. 
Figure 1E), and myeloid cells were more numerous in smoke-exposed mice compared to the control group (Suppl. Figure 1E). These data indicate that smoke exposure differentiates bone marrow progenitors into hematopoietic cells. Furthermore, the data suggest a preferential egress of myeloid cells from the bone marrow after smoke exposure.

To further delineate the contributions of hypoxia in systemic inflammation, we used a mouse model of chronic hypoxia. We quantified inflammatory cytokines and leukocytes in mice exposed to hypoxic conditions compared to normoxic controls. We observed increased expression of $\mathrm{Il}$ $1 b, I l-6, I l-18$ and Tnfa in the lungs of hypoxic mice (Figure 2A). Consistent with this heightened expression of the inflammatory cytokine genes, we found elevated concentrations of Il-6 in peripheral blood and lung parenchyma (Figure 2B). Furthermore, we found that mice in chronic hypoxic conditions had increased numbers of monocytes, neutrophils and lymphocytes in the blood compared to normoxic control mice (Figure 2C and Suppl. Figure 2A and B). Similarly, the lungs of hypoxic mice harbored augmented numbers of inflammatory cells, including interstitial macrophages (Figure 2D and Suppl. Figure 2C), which are reported to have pathologic roles in pulmonary diseases characterized by hypoxia ${ }^{48-50}$. Altogether, these data demonstrate leukocytosis in patients and mice with hypoxia.

Hypoxia increases proliferation of hematopoietic stem and progenitor cells.

Increased leukocyte numbers in the blood of hypoxic mice may indicate two underlying mechanisms: A) accelerated egress of bone marrow leukocyte into the blood and B) escalated production of leukocytes by hematopoietic stem and progenitor cells (HSPC) in the bone marrow. We found that although hypoxia increased bone marrow leukocyte numbers, there was no 
significant difference in the ratios of blood and bone marrow leukocyte numbers between normoxic and hypoxic mice (Suppl. Figure 2D), suggesting that egress of leukocytes from the bone marrow is unaltered in hypoxic conditions. To determine whether the observed hypoxiainduced leukocytosis represents increased production of mature leukocytes, we assessed the number and proliferation of bone marrow HSPC, which consist of hematopoietic stem cells (HSC), lineage ${ }^{-} \mathrm{Sca}-1^{+} \mathrm{c}-\mathrm{Kit}^{+}$(LSK or HSPC) cells and granulocyte macrophage progenitors (GMP) (Suppl. Figure 3). Flow cytometry analysis revealed that hypoxia expanded the numbers and frequencies of these cells in the femur (Figure 3A). Confocal microscopy confirmed a higher density of HSPC in the bone marrow of hypoxic mice compared to the normoxic control (Figure 3B). In line with augmented leukocyte and HSPC numbers, mice in hypoxic conditions had higher proportions of proliferating HSPC (Figure 3C-E). Additionally, we isolated HSPC and GMPs from mice kept under either normoxic or hypoxic conditions for three weeks. qPCR for the cell cycle genes revealed that the progenitors expressed higher levels of these genes in hypoxic conditions (Suppl. Figure 4A). HSPC proliferation is prerequisite for their differentiation into leukocytes. To understand if HSPC expansion in hypoxic conditions could result in their higher differentiation, we analyzed a whole genome transcriptome data comparing human $\mathrm{CD} 34^{+} \mathrm{HSPC}$ cultured under hypoxic vs. normoxic conditions ${ }^{51}$. A pathway analysis revealed that the genes involved in differentiation of stem cell, cell cycle and stimulation of cells were enriched in HSPC in hypoxic conditions (Suppl. Figures 4B-7). To discern if hypoxia accelerates HSPC differentiation, we adoptively transferred $\mathrm{GFP}^{+} \mathrm{HSPC}$ into mice housed under normoxic and hypoxic conditions, and enumerated progenies by flow cytometry. The bone marrow, blood and lungs of hypoxic mice contained augmented percentages of donor-derived differentiated leukocytes, lymphocytes, monocytes and neutrophils (Figure 3F and Suppl. Figure 8A). This 
difference of unleashed leukocyte production could not be attributed to higher engraftment of $\mathrm{GFP}^{+}$HSPC under hypoxia (Suppl. Figure 8B). In summary, these data indicate that hypoxia drives HSPC into the cell cycle and increases their differentiation into inflammatory cells.

VEGFr expression is increased in progenitor cells following hypoxia exposure and in a COPD mouse model.

The role of VEGF/ VEGFr in hypoxia-induced angiogenesis is well-documented ${ }^{33,52,53}$. To investigate the possible role of VEGF-A/VEGFr in hypoxia-mediated HSPC proliferation, we first assessed RNA expression of these genes in the whole bone marrow of hypoxic mice. The expression of $V e g f a$, but not $V e g f r$, was increased in bone marrow cells of hypoxic mice compared to their normoxic counterparts (Figure 4A). Interestingly, the expression of Vegfr mRNA and protein in bone marrow HSC and GMP increased in hypoxia (Figure 4B \& 4C). In line with these results, in a mouse model of COPD, we observed that mice exposed to cigarette smoke harbored higher frequency of Vegfr ${ }^{+}$HSPC and GMP (Suppl. Figure 9A). VEGFR expression in the progenitors also increased (Suppl. Figure 9B). Next, we identified the genes downstream to the VEGFr signaling using Ingenuity Pathway Analysis (Suppl. Figure 9C). The expression of these genes was elevated in HSPC cultured under hypoxic conditions (Figure 4D). These data indicate that hypoxia increases VEGFr signaling in HSPC. Hypoxia-inducible factor $1 \alpha(\mathrm{HIF}-1 \alpha)$, a key transcription factor increased in hypoxia, binds to several genes, and increases their expression ${ }^{54-}$ 57. We observed increased Hifla expression (Suppl. Figure 9D) in BM HSPC and GMP of hypoxic mice. Flow cytometry (Suppl. Figure 9E) revealed increased numbers of Hif-1 $\alpha^{+}$HSPC and GMP in hypoxic mice. Additionally, we confirmed decreased expression of prolyl-2 hydroxylase, which accelerates ubiquitination and lysosomal degradation of Hif- $1 \alpha$, in HSPC of 
hypoxic mice (Suppl. Figure 9F). Corresponding to the fact that Hif-1 $\alpha$ increases glycolysis, we observed elevated expression of glycolytic genes in bone marrow HSPC of hypoxic mice (Suppl. Figure 9G). To decipher the mechanisms of increased Vegfr expression in hypoxia, we ascertained if the VEGFR promoter region has HIF-1 $\alpha$ binding sites. Our in silico analysis revealed that HIF$1 \alpha$ has several binding sites on the $V E G F r$ promoters in both humans and mice (Figure $4 \mathbf{E}$ ). Chromatin immunoprecipitation experiments confirmed that HIF-1 $\alpha$ binds to the Vegfr promoter in mouse HSPC (Figure 4F). HIF-1 $\alpha$ inhibition significantly diminished hypoxia-mediated expression of $V E G F r$ in human (Figure 4G) and mouse (Figure 4H) HSPC. To delineate the role of HIF-1 $\alpha$ in hypoxia-induced HSPC proliferation, we silenced this transcription factor in mouse HSPC and observed significant downregulation of the genes involved in cell cycle progression (Figure 4I) as well as the genes downstream to the VEGFr signaling (Suppl. Figure 9H). In aggregate, these data indicate that HIF- $1 \alpha$ directly binds to the $V E G F r$ promoter in HSPC, increases $V E G F r$ expression and augments hematopoietic progenitor proliferation under hypoxic conditions.

\section{VEGFr inhibition decreases HSPC proliferation.}

The role of the VEGF signaling is well documented in angiogenesis in different diseases such as cancer and age-related macular degeneration ${ }^{27-29,53,58}$. However, the contribution of VEGFr in HSPC proliferation has not been studied. To understand the role of VEGFr in hypoxia-mediated HSPC proliferation, we cultured HSPC sorted from mouse bone marrow with Sugen 5416, a VEGFr inhibitor, under hypoxic and normoxic conditions. VEGFr inhibition significantly decreased the expression of cell cycle genes responsible for HSPC proliferation (Figure 5A and Suppl. Figure 10). To further examine the role of VEGFr signaling in proliferation of HSPC, we assessed proliferation of GMP in hypoxic mice injected with the VEGFr inhibitor. Compared to 
untreated controls, VEGFr inhibitor-injected mice had lower proportions of GMPs in the S-G2-M cell cycles (Figure 5B \& 5C). These mice had increased numbers of quiescent cells in the G0 phase. Interestingly, VEGFr inhibition also increased the frequency of GMP in the G1 phase, indicating a G1 to S-G2M arrest in the absence of VEGFr signaling. Additionally, there was a significant reduction in GMP numbers in the bone marrow of hypoxic mice after VEGFr inhibition (Figure 5D).

VEGFr inhibition decreased inflammation and inflammatory cell numbers.

We wanted to evaluate whether the decreased proliferation of HSPC after VEGFr inhibition would have an impact on inflammatory leukocyte generation. To this end, we differentiated HSPC isolated from mouse bone marrow in presence of the VEGFr inhibitor in hypoxic and normoxic conditions. VEGFr inhibition in hypoxic and normoxic HSPC significantly suppressed their differentiation into lymphoid cells, myeloid cells, B cells, monocytes and neutrophils (Figure 6A and Suppl. Figure 11A). Next, we investigated if VEGFr inhibition decreases hypoxia-induced leukocytosis in vivo. Compared to untreated control mice, VEGFr inhibitor-treated mice had decreased percentages and numbers of monocytes and neutrophils in peripheral blood and bone marrow (Suppl. Figure 11B and C and Figure 6B \& 6C). Additionally, we utilize an additional loss of function approach since Sugen is selective rather than specific for VEGFR2 and also inhibits PDGFR, KIT, RET, FLT-3, ABL and ALK. We silenced Vegfr2 in sorted murine HSPC placed in hypoxia for 24 hours. We found that the ability of HSPC to differentiate into mature leukocytes, especially B cells, myeloid cells, monocytes and neutrophils, was reduced in HSPC treated with siVegfr compared to siCtl (Suppl. Figure 12A). Additionally, we found decreased expression of cell cycle check point genes such as Cdk3, Cdk4, and Mki67 after Vegfr knock down 
(Suppl. Figure 12B). Overall, these data indicate the importance of VEGFr in the proliferation and differentiation of HSPC into leukocytes under hypoxic conditions. 


\section{Discussion}

Patients with hypoxic lung disease, including COPD and OSA, demonstrate chronic inflammation evidenced by elevated inflammatory cytokine levels and inflammatory leukocyte numbers. The degree of systemic inflammation portends clinical outcomes of hypoxic lung disease as well as severity of comorbid conditions $\mathbf{s}^{7,12,42,59,60}$. There is growing evidence that correction of underlying hypoxia-for example with CPAP in OSA-can reduce systemic inflammation highlighting the importance of hypoxia in inflammation ${ }^{61}$.

Consistent with serum cytokine levels observed in COPD and OSA, we observed increased levels of IL-1b, IL-6, IL-18, and TNF-a in peripheral blood of mice exposed to hypoxia. Both TNF-a and IL-6 have been shown to be independent risk factors of increased morbidity and mortality in hypoxic lung disease, particularly in COPD ${ }^{62}$. IL-6 has been shown to promote proliferation of HSC ${ }^{63}$, but interestingly, TNF- $\alpha$ has exhibited a contrasting function regulating hematopoiesis ${ }^{64}$. Systemic inflammation has long been postulated to drive the pathogenesis of diseases such as atherosclerosis. The CANTOS trial, assessing monoclonal antibody inhibition of IL-1b, has clearly demonstrated a benefit of attenuating inflammation independent of cholesterol lowering therapies in atherosclerosis ${ }^{65}$. Together, these findings illustrate an important role of inflammation in hypoxic lung disease-associated comorbidities.

Elevated numbers of leukocytes in peripheral blood has received recent attention as a marker of inflammation and independent risk factor for diseases such as atherosclerosis ${ }^{66,67}$ and diabetes ${ }^{68}$. Lodge et al. illustrated the role of neutrophil-mediated lung damage in patients with COPD ${ }^{69}$. 
Similarly, neutrophilia has been demonstrated as a key feature of obstructive sleep apnea and postulated to contribute to the pathogenesis of $\mathrm{OSA}^{70}$. The present study demonstrates a mechanism of hypoxia-mediated expansion of inflammatory leukocytes, which likely contribute to the pathogenesis and comorbid conditions of hypoxic lung diseases.

In response to tissue injury, mature leukocytes may be mobilized from sequestered sites or generated de novo from hematopoietic tissues ${ }^{71,72}$. It is reasonable to postulate acute increase in recruitment of mature leukocytes from hematopoietic sites contributes to a relative paucity of leukocytes in the bone marrow and abundance in peripheral circulation. In contrast, under chronic inflammation, expansion of HSPC maintains reservoirs of leukocytes in hematopoietic tissues ${ }^{73,74}$. In line with this, we observed a symmetric expansion of leukocytes in the peripheral blood and bone marrow in hypoxic conditions.

VEGFr signaling in the proliferation and differentiation of endothelial cells in angiogenesis, particularly in the development of cancer ${ }^{75,76}$, has been well documented. However, the role of VEGF signaling in the proliferation of hematopoietic stem cells and hematopoiesis has not been studied. Our study demonstrates that the proliferation of HSPC and leukocytosis under hypoxic conditions is mediated by VEGFr. Thus, the current study identifies VEGFr as a novel target to dampen inflammation in diseases characterized by hypoxia. Randomized clinical trials will be required to validate the therapeutic efficacy of this target.

HIF-1 $\alpha$ is an important regulator of VEGFA expression in local hypoxia, such as tumor microenvironment ${ }^{77}$. However, VEGFr regulation by HIF-1 $\alpha$ is not known. Our in silico and 
molecular experiments demonstrated that HIF- $1 \alpha$ directly interacts with the $V E G F r$ promoters in human and mouse HSPC under hypoxic conditions contributing to increased expression of this receptor. The importance of this finding was further supported by suppression of HSPC proliferation and leukocyte differentiation after Hifla silencing in HSPC. However, we acknowledge that we do not know if higher HSPC differentiation is due to the direct effect of hypoxia/ HIF-1 $\alpha$ or the resulting inflammation. Although our data show that Hifla silencing decreased HSPC proliferation (Figure 4I), HIF-1 $\alpha$ also promotes inflammation. Congruently, our and other groups have shown that hypoxia results in systemic inflammation ${ }^{78-80}$. Proinflammatory cytokines can drive HSPC into the cell cycle and increase their proliferation. Thus, exaggerated inflammation may increase lineage output from HSPC in hypoxic mice. Future studies are warranted to understand these mechanisms to reveal potential molecular targets to reduce inflammation in hypoxic diseases. 


\section{Methods}

Animals

All animal experiments were conducted following NIH and ARRIVE guidelines under protocols approved by the Institutional Animal Care and Use Committee of the University of Pittsburgh. Ten to twelve-week-old C57BL/6J male mice were exposed to normobaric hypoxic-10\% oxygen, which represents $50 \%$ of the normal amount of oxygen, or normoxic conditions for 3 weeks. This oxygen concentration resulted in increased right ventricular systolic pressure, Fulton index and pulmonary vascular remodeling ${ }^{80,81}$. Of note, we have observed a very high mortality when we further decreased $\mathrm{O}_{2}$ concentration. Aftyer 21 days in hypoxic chamber, mice underwent right ventricular catheterization, followed by tissue and blood collection. The mice were anesthetized with ketamine/xylazine and ventilated through a transtracheal catheter.

\section{Flow Cytometry}

C57BL/6 mice were anesthetized, and a small volume of peripheral blood was collected by cardiac puncture, followed by transcardial perfusion with $15 \mathrm{~mL}$ of ice-cold PBS. One lobe of the lung was harvested, minced and digested with collagenase I, collagenase XI, and hyaluronidase for one hour at 37C. After incubation, a single cell suspension was prepared by passing the digested lung tissue through a $70 \mu \mathrm{m}$ nylon strainer, followed by washing with $0.5 \%$ bovine serum albumin in PBS (FACS buffer). The filtrate was washed with $10 \mathrm{~mL}$ of FACS buffer and centrifuged at $4{ }^{\circ} \mathrm{C}$ for 7 minutes at $350 \mathrm{~g}$. The supernatant was discarded, and the samples were re-suspended and labeled with 600 -fold diluted antibody mixture with the following antibodies: anti-CD45.2 (BD Biosciences clone 104, cat\#560693), CD11b (BD Biosciences, clone M1/70, cat\#557657), CD115 (BD Biosciences, clone T38-320, cat\# 565249), Ly6G (BD Biosciences, clone 1A8, cat\#564979) 
and samples were incubated on ice for 1 hour. A Fortessa Flow Cytometer (BD) was used to acquire data. Data were analyzed with FlowJo software (Tree Star).

\section{Biochemical Assays}

Concentrations of cytokines in plasma and parenchymal tissues were quantified by enzyme-linked immunosorbent assay (ELISA). IL-6 levels were quantified in lung tissue from hypoxic and normoxic conditions using a mouse IL-6 capture ELISA kit following the manufacturer instructions (Invitrogen, Cat \# BMS603-2). Comparisons between conditions was performed using Graphpad Prism, and absolute concentration was calculated based on a lyophilized standard IL-6 included in the kit.

\section{RT PCR}

Lung and peripheral blood samples were harvested and immediately snap frozen in liquid nitrogen until they could be processed. RNA extraction was performed using the PicoPure RNA isolation kit (Applied BioSystems, Cat \# KIT0204) without modification to the manufacturer protocol. Total RNA was quantified using a NanoDrop spectrophotometer. Complementary DNA was generated from $100 \mathrm{ng}$ of mRNA per sample using the high capacity cDNA Reverse Transcription kit (Applied BioSystems, Cat \# 4368814). Relative gene expression was determined by qPCR with the PowerUp SYBR Green reporter (Applied BioSystems, Cat \# A25742) and primers supplied by IDT; gene expression was represented as $\Delta \mathrm{Ct}$ normalized to beta-actin expression.

\section{Whole Mount Imaging}

Freshly dissected sternums from C57BL/6 mice exposed to normoxic or hypoxic conditions were fixed in cold 4\% paraformaldehyde (PFA, ThermoFisher Scientific, Cat \# 28908) in PBS for 25 minutes without agitation. The bones were washed in PBS three times with 15-minute incubations before incubating with common lineage biotinylated antibodies, including anti-TER119 
(Biolegend, clone TER-119, cat\#116204), CD11b (BD Biosciences, clone M1/70, cat\#553309), CD11c (Biolegend, clone N418, cat\#117304), CD45R/B220 (Biolegend, clone RA3-6B2, cat\#103204), NK1.1 (Biolegend, clone PK136, cat\#108704), GR-1 (Biolegend, clone RB6-8C5, cat\#108404), CD4 (Biolegend, clone GK1.5, cat\#100404), CD8 (Biolegend, clone 53-6.7, cat\#100704) and CD127 (Biolegend, clone A7R34, cat\#135006). Samples were incubated for 24 hours at $4 \mathrm{C}$, then washed three times with $1 \%$ FBS in PBS with 15-minute incubations, then labeled with Streptavidin FITC antibody, followed by overnight incubation at $4^{\circ} \mathrm{C}$. The samples were sequentially labeled with anti-VE Cadherin (BD Biosciences, clone 11D4.1, cat\# 562242) and anti-CD31 APC antibodies (BD Biosciences, clone MEC 13.3, cat\# 551262), followed by rabbit anti-mouse CD150 then rat anti-rabbit Cy3 (Invitrogen) with three wash steps between primary and secondary antibody overnight incubations. Lastly, the samples were washed three times and imaged on a Nikon A1 confocal microscope; images were analyzed using ImageJ software.

\section{Human Whole Blood data}

All human data were collected in accordance with a University of Pittsburgh Independent Review Board approved protocol. For each sample collected, an informed consent was obtained from all subjects and/or their legal guardian(s). Patients with intermittent hypoxia, defined by an Apnea hypoxia index $(\mathrm{AHI})>5$, were eligible for inclusion in the obstructive sleep apnea group, and matched controls with AHI less than 5 were eligible for inclusion in the control group. Patients with pulmonary function testing (PFT) confirmed chronic obstructive lung disease were eligible for inclusion in the COPD group. Patients with concurrent conditions known to cause leukocytosis were excluded, including known history of CVD, dyslipidemia, diabetes mellitus, allergic, pulmonary or hematological disease, malignancy, recent injury or surgery, recent systemic steroid 
use, current infection or any systemic medication use (e.g. hypolipidemic or anti-platelet agents). Complete blood cell count laboratory data were collected prior to medical interventions. HSPC Cell Culture

Hematopoietic stem and progenitor cells were isolated by negative magnetic-activated cell sorting from vertebral, femoral and tibial bone marrow (StemCell Technologies, Cat \# 17665). Cells were cultured in the presence of G-CSF on 96 well round-bottom cell culture plates with 10, 50, or 100 nM concentrations of VEGFr inhibitor, Sugen (SU5416, MedChem Express), or with siRNA control (siCtl, IDT) or against $V e g f r$ (siVegfr, IDT), for 72 hours prior to hypoxia challenge. Samples were exposed to normobaric hypoxic conditions $\left(10 \% \mathrm{O}_{2}\right)$ for 24 hours prior to RNA extraction and flow cytometry analysis.

\section{RNA Sequencing}

Publicly available differentially expressed genes from HSPC exposed to hypoxic conditions were assessed for genes of interest including VEFGA and VEGFr and heatmap representations of the data were prepared. Enriched pathways were discerned using Ingenuity Pathway Analysis.

\section{ChIP Sequencing}

To assess the interaction between HIF-1a and VEGFr, we first located the promoter region of VEGFr using the UCSC genome browser (University of California Santa Cruz). Then, we looked for potential HIF-1-alpha binding sites onto the promoter region of VEGFr using Transfac software (GeneXplain). The primers for each HIF-1 $\alpha$ binding site were designed using NCBI PrimerBLAST. Murine HSPC were harvested from the bone marrow of C57BL/6 mice. Cells were resuspended in complete SFEM media and plated onto $10 \mathrm{~cm}$ dishes. Cells were incubated for 24 hours in normoxia or hypoxia. Cells were then harvested and resuspended in PBS at a concentration $10^{6}$ cells/ mL. Cells were then fixed, and DNA was extracted and 
immunoprecipitated by a ChIP grade HIF-1 $\alpha$ antibody (Rb polyclonal, Novusbio, Cat \# NB100479SS) as previously described ${ }^{82}$. Finally, real time PCR was run to quantify the amount of HIF $1 A$ bound to $V E G F r$ promoter region in each condition.

Adoptive transfer experiment

C57BL/6 mice were placed under either normoxic or hypoxic conditions $\left(10 \% \mathrm{O}_{2}\right)$ for 10 days. At day 7 of hypoxia, bone marrow $\mathrm{HSPC}$ from $\mathrm{KIkGR}^{+}$mice were negatively enriched using a magnetic separation method and retro-orbitally injected into either normoxic or hypoxic C57BL/6 mice at a concentration of 20 million cell $/ \mathrm{mL}$. Mice were sacrificed, and lungs, bone marrow, spleen and blood were harvested at day 10 of hypoxia. The percentages of progenies derived from the adoptively transferred cells were measured by flow cytometry. 


\section{Acknowledgments:}

This work was supported by National Institute of Health grants R00HL121076-03, R01HL143967, and R01HL142629, AHA Transformational Project Award (19TPA34910142), AHA Innovative Project Award (19IPLOI34760566) and ALA Innovation Project Award (IA-629694) (to PD); NIH grants R01 HL124021, HL 122596, HL 138437, and UH2/UH3 TR002073 as well as the American Heart Association Established Investigator Award 18EIA33900027 (to S.Y.C.); and the VMI Postdoctoral Training Program in Translational Research and Entrepreneurship in Pulmonary and Vascular Biology T32 funded by the National, Heart, Lung and Blood Institute (NHLBI) (to J. F.). We thank the NIH supported microscopy resources in the Center for Biologic Imaging (NIH grant 1S10OD019973-01). We thank The Center for Organ Recovery \& Education (CORE) as well as organ donors and their families for the generous donation of tissues used in this study.

\section{Disclosure:}

S.Y.C. has served as a consultant for Zogenix, Aerpio, and United Therapeutics; S.Y.C. holds research grants from Actelion and Pfizer. S.Y.C. has filed patent applications regarding the targeting of metabolism in pulmonary hypertension. The authors declare no other conflicts of interest.

\section{Author Contributions}

J.F and S.P.O conducted experiments, data analysis, and wrote the manuscript. S.B.., L.L.O and A.A conducted experiments. S.G. and S.F.O helped with the $\mathrm{SpO}_{2}$ measurement experiment. C.R.K, S.G, J.C.B and J.S helped with the experiments involving the mouse model of COPD. S.Y.C reviewed the manuscript and provided us with hypoxic chambers for culturing HSCPC. P.D designed the research study and composed the manuscript. 


\section{References:}

Data Availability Statement: For data sharing and information please contact Dr. Partha Dutta, the corresponding author at duttapa@pitt.edu.

1 Maselli, D. J. et al. Clinical Epidemiology of COPD: Insights From 10 Years of the COPDGene Study. Chest 156, 228-238, doi:https://doi.org/10.1016/i.chest.2019.04.135 (2019).

2 Brat, K. et al. Respiratory parameters predict poor outcome in COPD patients, category GOLD 2017 B. Int J Chron Obstruct Pulmon Dis 13, 1037-1052, doi:10.2147/COPD.S147262 (2018).

3 Abuzaid, A. S. et al. Meta-Analysis of Cardiovascular Outcomes With Continuous Positive Airway Pressure Therapy in Patients With Obstructive Sleep Apnea. Am J Cardiol 120, 693699, doi:10.1016/j.amjcard.2017.05.042 (2017).

4 Geovanini, G. R. et al. Association between Obstructive Sleep Apnea and Cardiovascular Risk Factors: Variation by Age, Sex, and Race. The Multi-Ethnic Study of Atherosclerosis. Ann Am Thorac Soc 15, 970-977, doi:10.1513/AnnalsATS.201802-1210C (2018).

5 Song, S. O. et al. Metabolic Consequences of Obstructive Sleep Apnea Especially Pertaining to Diabetes Mellitus and Insulin Sensitivity. Diabetes Metab J 43, 144-155, doi:10.4093/dmj.2018.0256 (2019).

6 Yaggi, H. K. et al. Reducing cardiovascular risk through treatment of obstructive sleep apnea: 2 methodological approaches. Am Heart J 172, 135-143, doi:10.1016/j.ahj.2015.07.033 (2016).

7 Vujic, T., Nagorni, O., Maric, G., Popovic, L. \& Jankovic, J. Metabolic syndrome in patients with chronic obstructive pulmonary disease: frequency and relationship with systemic inflammation. Hippokratia 20, 110-114 (2016).

8 Chen, W., Thomas, J., Sadatsafavi, M. \& FitzGerald, J. M. Risk of cardiovascular comorbidity in patients with chronic obstructive pulmonary disease: a systematic review and meta-analysis. Lancet Respir Med 3, 631-639, doi:10.1016/S2213-2600(15)00241-6 (2015).

9 Cubillos-Zapata, C. et al. Obstructive Sleep Apnea Monocytes Exhibit High Levels of Vascular Endothelial Growth Factor Secretion, Augmenting Tumor Progression. Mediators Inflamm 2018, 7373921, doi:10.1155/2018/7373921 (2018).

10 Chao, J., Wood, J. G. \& Gonzalez, N. C. Alveolar macrophages initiate the systemic microvascular inflammatory response to alveolar hypoxia. Respir Physiol Neurobiol 178, 439-448, doi:10.1016/j.resp.2011.03.008 (2011).

11 Chao, J., Wood, J. G., Blanco, V. G. \& Gonzalez, N. C. The systemic inflammation of alveolar hypoxia is initiated by alveolar macrophage-borne mediator(s). Am J Respir Cell Mol Biol 41, 573-582, doi:10.1165/rcmb.2008-04170C (2009).

12 Kheirandish-Gozal, L. \& Gozal, D. Obstructive Sleep Apnea and Inflammation: Proof of Concept Based on Two Illustrative Cytokines. Int J Mol Sci 20, doi:10.3390/ijms20030459 (2019). 
13 Gonzalez, N. C. \& Wood, J. G. Alveolar hypoxia-induced systemic inflammation: what low PO(2) does and does not do. Adv Exp Med Biol 662, 27-32, doi:10.1007/978-1-4419-12411_3 (2010).

14 Lopez-Campos, J. L. et al. Implications of the inflammatory response for the identification of biomarkers of chronic obstructive pulmonary disease. Biomark Med 10, 109-122, doi:10.2217/bmm.15.87 (2016).

15 MacNee, W. Systemic inflammatory biomarkers and co-morbidities of chronic obstructive pulmonary disease. Ann Med 45, 291-300, doi:10.3109/07853890.2012.732703 (2013).

16 Yokoe, T. et al. Elevated levels of C-reactive protein and interleukin- 6 in patients with obstructive sleep apnea syndrome are decreased by nasal continuous positive airway pressure. Circulation 107, 1129-1134, doi:10.1161/01.cir.0000052627.99976.18 (2003).

17 Schulz, R. et al. Enhanced release of superoxide from polymorphonuclear neutrophils in obstructive sleep apnea. Impact of continuous positive airway pressure therapy. Am J Respir Crit Care Med 162, 566-570, doi:10.1164/ajrccm.162.2.9908091 (2000).

18 Andrade, D. R. et al. Oxygen supplementation increases the total work and muscle damage markers but reduces the inflammatory response in COPD patients. Respiratory Physiology \& Neurobiology 280, 103475, doi:https://doi.org/10.1016/i.resp.2020.103475 (2020).

19 de Winther, M. P. J. \& Lutgens, E. The Link between Hematopoiesis and Atherosclerosis. N Engl J Med 380, 1869-1871, doi:10.1056/NEJMcibr1901397 (2019).

20 Mo, L. et al. Severe obstructive sleep apnea is associated with significant coronary artery plaque burden independent of traditional cardiovascular risk factors. Int J Cardiovasc Imaging 36, 347-355, doi:10.1007/s10554-019-01710-w (2020).

21 Murphy, A. M. et al. Intermittent hypoxia in obstructive sleep apnoea mediates insulin resistance through adipose tissue inflammation. Eur Respir J 49, doi:10.1183/13993003.01731-2016 (2017).

22 Baldridge, M. T., King, K. Y., Boles, N. C., Weksberg, D. C. \& Goodell, M. A. Quiescent haematopoietic stem cells are activated by IFN-gamma in response to chronic infection. Nature 465, 793-797, doi:10.1038/nature09135 (2010).

23 Shi, C. et al. Bone marrow mesenchymal stem and progenitor cells induce monocyte emigration in response to circulating toll-like receptor ligands. Immunity 34, 590-601, doi:10.1016/j.immuni.2011.02.016 (2011).

24 Heidt, T. et al. Differential contribution of monocytes to heart macrophages in steadystate and after myocardial infarction. Circ Res 115, 284-295, doi:10.1161/CIRCRESAHA.115.303567 (2014).

25 Zigmond, E. et al. Ly6C hi monocytes in the inflamed colon give rise to proinflammatory effector cells and migratory antigen-presenting cells. Immunity 37, 1076-1090, doi:10.1016/j.immuni.2012.08.026 (2012).

26 Valipour, A. et al. Circulating vascular endothelial growth factor and systemic inflammatory markers in patients with stable and exacerbated chronic obstructive pulmonary disease. Clin Sci (Lond) 115, 225-232, doi:10.1042/CS20070382 (2008).

27 Yang, F. et al. Association between VEGF Gene Polymorphisms and the Susceptibility to Lung Cancer: An Updated Meta-Analysis. Biomed Res Int 2018, 9271215, doi:10.1155/2018/9271215 (2018). 
28 Zong, S. et al. Prognostic significance of VEGF-C immunohistochemical expression in colorectal cancer: A meta-analysis. Clin Chim Acta 458, 106-114, doi:10.1016/j.cca.2016.04.037 (2016).

29 Liu, W., Dong, Z., Hu, R. \& Wang, C. Association of Vascular Endothelial Growth Factor ( VEGF) Gene Polymorphisms With Gastric Cancer and Its Development, Prognosis, and Survival. Technol Cancer Res Treat 17, 1533034617753810, doi:10.1177/1533034617753810 (2018).

30 Archontogeorgis, K. et al. Serum Levels of Vascular Endothelial Growth Factor and Insulinlike Growth Factor Binding Protein-3 in Obstructive Sleep Apnea Patients: Effect of Continuous Positive Airway Pressure Treatment. Open Cardiovasc Med J 9, 133-138, doi:10.2174/1874192401509010133 (2015).

31 Baz-Davila, R. et al. Role of HIF1A, VEGFA and VEGFR2 SNPs in the Susceptibility and Progression of COPD in a Spanish Population. PLoS One 11, e0154998, doi:10.1371/journal.pone.0154998 (2016).

32 Hwangbo, C. et al. Modulation of Endothelial Bone Morphogenetic Protein Receptor Type 2 Activity by Vascular Endothelial Growth Factor Receptor 3 in Pulmonary Arterial Hypertension. Circulation 135, 2288-2298, doi:10.1161/CIRCULATIONAHA.116.025390 (2017).

33 Ramakrishnan, S., Anand, V. \& Roy, S. Vascular endothelial growth factor signaling in hypoxia and inflammation. J Neuroimmune Pharmacol 9, 142-160, doi:10.1007/s11481014-9531-7 (2014).

$34 \mathrm{Fu}, \mathrm{X}$. \& Zhang, F. Role of the HIF-1 signaling pathway in chronic obstructive pulmonary disease. Exp Ther Med 16, 4553-4561, doi:10.3892/etm.2018.6785 (2018).

35 Farid Hosseini, R. et al. Serum levels of vascular endothelial growth factor in chronic obstructive pulmonary disease. Med J Islam Repub Iran 28, 85 (2014).

36 Gomez-Arroyo, J. et al. A brief overview of mouse models of pulmonary arterial hypertension: problems and prospects. Am J Physiol Lung Cell Mol Physiol 302, L977-991, doi:10.1152/ajplung.00362.2011 (2012).

37 Vitali, S. H. et al. The Sugen 5416/hypoxia mouse model of pulmonary hypertension revisited: long-term follow-up. Pulmonary circulation 4, 619-629, doi:10.1086/678508 (2014).

38 Stenmark, K. R., Meyrick, B., Galie, N., Mooi, W. J. \& McMurtry, I. F. Animal models of pulmonary arterial hypertension: the hope for etiological discovery and pharmacological cure. American Journal of Physiology-Lung Cellular and Molecular Physiology 297, L1013L1032, doi:10.1152/ajplung.00217.2009 (2009).

39 Kliment, C. R. et al. Adenine nucleotide translocase regulates airway epithelial metabolism, surface hydration and ciliary function. Journal of Cell Science 134, doi:10.1242/jcs.257162 (2021).

40 Hautamaki, R. D., Kobayashi, D. K., Senior, R. M. \& Shapiro, S. D. Requirement for macrophage elastase for cigarette smoke-induced emphysema in mice. Science (New York, N.Y.) 277, 2002-2004, doi:10.1126/science.277.5334.2002 (1997).

41 Andrijevic, L., Milutinov, S., Andrijevic, I., Jokic, D. \& Vukoja, M. Association Between the Inflammatory Biomarkers and Left Ventricular Systolic Dysfunction in Patients with 
Exacerbations of Chronic Obstructive Pulmonary Disease. Balkan Med J 34, 226-231, doi:10.4274/balkanmedj.2016.1114 (2017).

42 Thomsen, M., Dahl, M., Lange, P., Vestbo, J. \& Nordestgaard, B. G. Inflammatory biomarkers and comorbidities in chronic obstructive pulmonary disease. Am J Respir Crit Care Med 186, 982-988, doi:10.1164/rccm.201206-11130C (2012).

43 Yona, S. et al. Fate mapping reveals origins and dynamics of monocytes and tissue macrophages under homeostasis. Immunity 38, 79-91, doi:10.1016/j.immuni.2012.12.001 (2013).

44 Soguel Schenkel, N., Burdet, L., de Muralt, B. \& Fitting, J. W. Oxygen saturation during daily activities in chronic obstructive pulmonary disease. Eur Respir J 9, 2584-2589, doi:10.1183/09031936.96.09122584 (1996).

45 Sliwinski, P., Lagosz, M., Gorecka, D. \& Zielinski, J. The adequacy of oxygenation in COPD patients undergoing long-term oxygen therapy assessed by pulse oximetry at home. European Respiratory Journal 7, 274-278 (1994).

46 Scott, A. S., Baltzan, M. A. \& Wolkove, N. Examination of pulse oximetry tracings to detect obstructive sleep apnea in patients with advanced chronic obstructive pulmonary disease. Canadian respiratory journal 21, 171-175, doi:10.1155/2014/948717 (2014).

47 Kliment, C. R. et al. Adenine nucleotide translocase regulates airway epithelial metabolism, surface hydration and ciliary function. Journal of cell science 134, doi:10.1242/jcs.257162 (2021).

48 Pugliese, S. C. et al. A Time- and Compartment-Specific Activation of Lung Macrophages in Hypoxic Pulmonary Hypertension. J Immunol 198, 4802-4812, doi:10.4049/jimmunol.1601692 (2017).

49 Tuleta, I. et al. Intermittent Hypoxia Contributes to the Lung Damage by Increased Oxidative Stress, Inflammation, and Disbalance in Protease/Antiprotease System. Lung 194, 1015-1020, doi:10.1007/s00408-016-9946-4 (2016).

50 Kojima, H. et al. Hypoxia-inducible factor-1 alpha deletion in myeloid lineage attenuates hypoxia-induced pulmonary hypertension. Physiol Rep 7, e14025, doi:10.14814/phy2.14025 (2019).

51 Wierenga, A. T., Vellenga, E. \& Schuringa, J. J. Convergence of hypoxia and TGF $\beta$ pathways on cell cycle regulation in human hematopoietic stem/progenitor cells. PLoS One 9, e93494, doi:10.1371/journal.pone.0093494 (2014).

52 Semenza, G. L. Regulation of hypoxia-induced angiogenesis: a chaperone escorts VEGF to the dance. The Journal of Clinical Investigation 108, 39-40, doi:10.1172/JCI13374 (2001).

53 Zhu, H. \& Zhang, S. Hypoxia inducible factor-1 $\alpha$ /vascular endothelial growth factor signaling activation correlates with response to radiotherapy and its inhibition reduces hypoxia-induced angiogenesis in lung cancer. Journal of Cellular Biochemistry 119, 77077718, doi:10.1002/jcb.27120 (2018).

54 Benita, Y. et al. An integrative genomics approach identifies Hypoxia Inducible Factor-1 (HIF-1)-target genes that form the core response to hypoxia. Nucleic acids research 37, 4587-4602, doi:10.1093/nar/gkp425 (2009).

55 Semenza, G. L. Hypoxia-inducible factors: mediators of cancer progression and targets for cancer therapy. Trends in pharmacological sciences 33, 207-214, doi:10.1016/j.tips.2012.01.005 (2012). 
56 Takeda, N. et al. Endothelial PAS domain protein 1 gene promotes angiogenesis through the transactivation of both vascular endothelial growth factor and its receptor, Flt-1. Circ Res 95, 146-153, doi:10.1161/01.RES.0000134920.10128.b4 (2004).

57 Mole, D. R. et al. Genome-wide association of hypoxia-inducible factor (HIF)-1alpha and HIF-2alpha DNA binding with expression profiling of hypoxia-inducible transcripts. The Journal of biological chemistry 284, 16767-16775, doi:10.1074/jbc.M901790200 (2009).

58 Gao, S., Ma, J. J. \& Lu, C. Prognostic significance of VEGF-C immunohistochemical expression in breast cancer: a meta-analysis. Tumour Biol 35, 1523-1529, doi:10.1007/s13277-013-1211-3 (2014).

59 Celli, B. R. et al. Inflammatory biomarkers improve clinical prediction of mortality in chronic obstructive pulmonary disease. Am J Respir Crit Care Med 185, 1065-1072, doi:10.1164/rccm.201110-17920C (2012).

60 Kelly, E., Owen, C. A., Pinto-Plata, V. \& Celli, B. R. The role of systemic inflammatory biomarkers to predict mortality in chronic obstructive pulmonary disease. Expert Rev Respir Med 7, 57-64, doi:10.1586/ers.12.82 (2013).

61 Perrini, S. et al. Correction of intermittent hypoxia reduces inflammation in obese subjects with obstructive sleep apnea. JCI Insight 2, doi:10.1172/jci.insight.94379 (2017).

62 Bradford, E. et al. The value of blood cytokines and chemokines in assessing COPD. Respir Res 18, 180, doi:10.1186/s12931-017-0662-2 (2017).

63 Tie, R. et al. Interleukin-6 signaling regulates hematopoietic stem cell emergence. Exp Mol Med 51, 1-12, doi:10.1038/s12276-019-0320-5 (2019).

64 Pronk, C. J., Veiby, O. P., Bryder, D. \& Jacobsen, S. E. Tumor necrosis factor restricts hematopoietic stem cell activity in mice: involvement of two distinct receptors. J Exp Med 208, 1563-1570, doi:10.1084/jem.20110752 (2011).

65 Ridker, P. M. Inflammation, cardiovascular disease and cancer: moving toward predictive medicine. CMAJ 189, E382-E383, doi:10.1503/cmaj.161033 (2017).

66 Kim, J. H., Lim, S., Park, K. S., Jang, H. C. \& Choi, S. H. Total and differential WBC counts are related with coronary artery atherosclerosis and increase the risk for cardiovascular disease in Koreans. PLoS One 12, e0180332, doi:10.1371/journal.pone.0180332 (2017).

67 Welsh, C. et al. Association of Total and Differential Leukocyte Counts With Cardiovascular Disease and Mortality in the UK Biobank. Arterioscler Thromb Vasc Biol 38, 1415-1423, doi:10.1161/ATVBAHA.118.310945 (2018).

68 Koo, H. K. et al. Systemic White Blood Cell Count as a Biomarker Associated with Severity of Chronic Obstructive Lung Disease. Tuberc Respir Dis (Seoul) 80, 304-310, doi:10.4046/trd.2017.80.3.304 (2017).

69 Lodge, K. M. et al. S114 Hypoxia drives neutrophil-mediated endothelial damage in copd. Thorax 72, A69, doi:10.1136/thoraxjnl-2017-210983.120 (2017).

70 Geovanini, G. R. et al. Elevations in neutrophils with obstructive sleep apnea: The MultiEthnic Study of Atherosclerosis (MESA). Int J Cardiol 257, 318-323, doi:10.1016/j.ijcard.2017.10.121 (2018).

71 Vasamsetti, S. B. et al. Sympathetic Neuronal Activation Triggers Myeloid Progenitor Proliferation and Differentiation. Immunity 49, 93-106.e107, doi:10.1016/j.immuni.2018.05.004 (2018). 
$72 \mathrm{Li}$, B. et al. Mobilized human hematopoietic stem/progenitor cells promote kidney repair after ischemia/reperfusion injury. Circulation 121, 2211-2220, doi:10.1161/circulationaha.109.928796 (2010).

73 Boettcher, S. \& Manz, M. G. Regulation of Inflammation- and Infection-Driven Hematopoiesis. Trends in immunology 38, 345-357, doi:10.1016/j.it.2017.01.004 (2017).

74 Fuchs, A. et al. Trauma Induces Emergency Hematopoiesis through IL-1/MyD88Dependent Production of G-CSF. The Journal of Immunology 202, 3020-3032, doi:10.4049/jimmunol.1801456 (2019).

75 Sui, $\mathrm{H}$. et al. Tanshinone IIA inhibits beta-catenin/VEGF-mediated angiogenesis by targeting TGF-beta1 in normoxic and HIF-1alpha in hypoxic microenvironments in human colorectal cancer. Cancer Lett 403, 86-97, doi:10.1016/j.canlet.2017.05.013 (2017).

76 Bao, B. et al. Hypoxia-induced aggressiveness of pancreatic cancer cells is due to increased expression of VEGF, IL-6 and miR-21, which can be attenuated by CDF treatment. PLoS One 7, e50165, doi:10.1371/journal.pone.0050165 (2012).

77 Chen, X. et al. Vascular endothelial growth factor (VEGF) regulation by hypoxia inducible factor-1 alpha (HIF1A) starts and peaks during endometrial breakdown, not repair, in a mouse menstrual-like model. Human Reproduction 30, 2160-2170, doi:10.1093/humrep/dev156 (2015).

78 Amsellem, V. et al. Roles for the CX3CL1/CX3CR1 and CCL2/CCR2 Chemokine Systems in Hypoxic Pulmonary Hypertension. American journal of respiratory cell and molecular biology 56, 597-608, doi:10.1165/rcmb.2016-02010C (2017).

79 Florentin, J. et al. Inflammatory Macrophage Expansion in Pulmonary Hypertension Depends upon Mobilization of Blood-Borne Monocytes. J Immunol 200, 3612-3625, doi:10.4049/jimmunol.1701287 (2018).

80 Florentin, J. et al. Interleukin-6 mediates neutrophil mobilization from bone marrow in pulmonary hypertension. Cellular \& molecular immunology 18, 374-384, doi:10.1038/s41423-020-00608-1 (2021).

81 Florentin, J. et al. Inflammatory Macrophage Expansion in Pulmonary Hypertension Depends upon Mobilization of Blood-Borne Monocytes. I Immunol, doi:10.4049/jimmunol.1701287 (2018).

82 Dahl, J. A. \& Collas, P. A rapid micro chromatin immunoprecipitation assay (ChIP). Nature Protocols 3, 1032-1045, doi:10.1038/nprot.2008.68 (2008). 


\section{Figure 1}

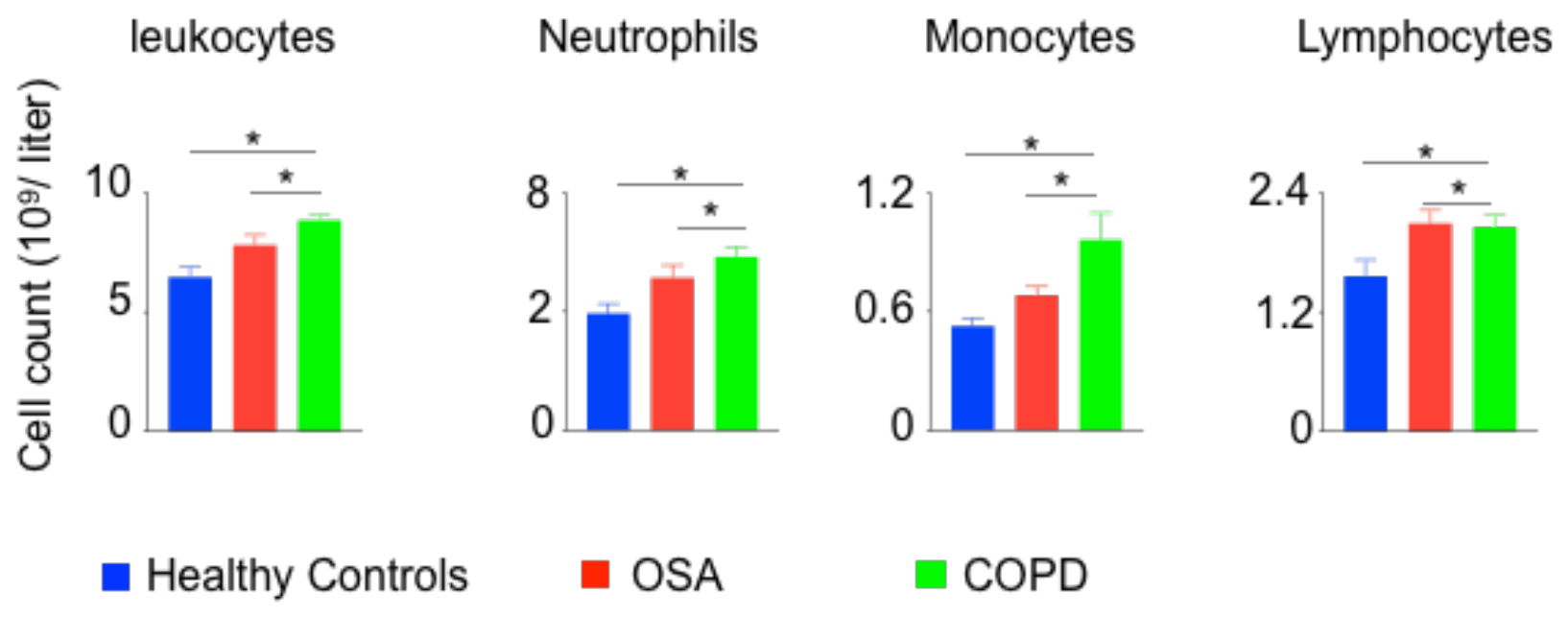

Figure 1: Patients with hypoxia have elevated numbers of inflammatory leukocytes.

A restrospective chart review was performed to enumerate inflammatory leukocytes in peripheral blood of patients with OSA and COPD. $n=26$ (OSA), 10 (COPD) and 22 (Healthy controls). Data are shown as mean \pm s.e.m. $* \mathrm{P}<0.05$. 
Figure 2

A

$\| 1 / b$

116

II18

Tnfa
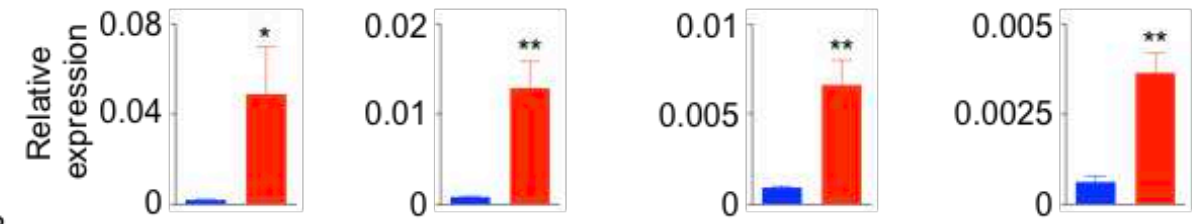

B

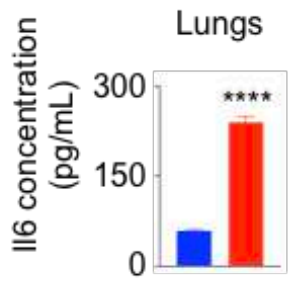

Blood

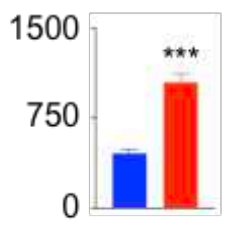

Normoxia

Hypoxia

C
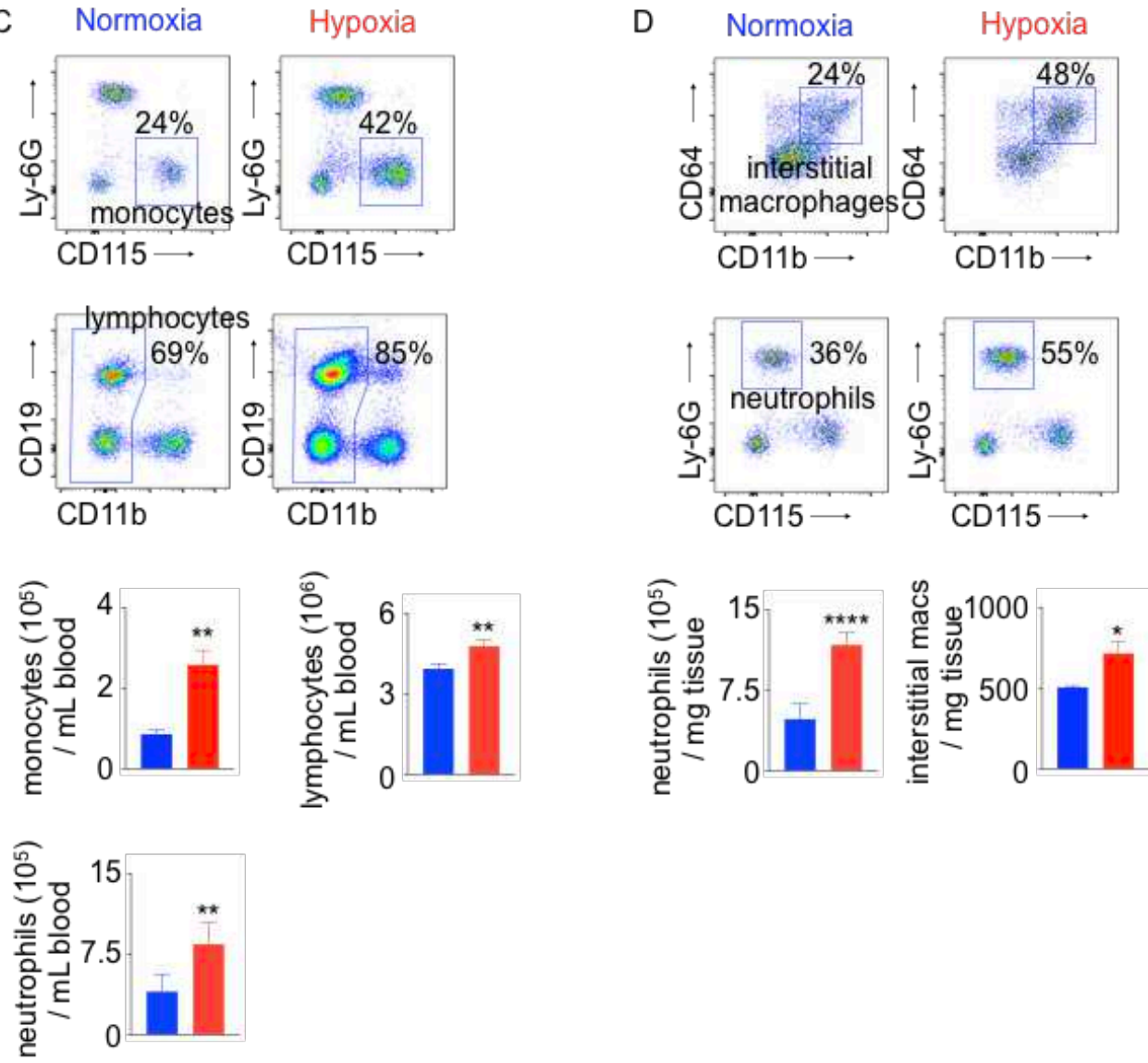

Figure 2: Hypoxia induces inflammation

C57BL/6 mice were placed under either normoxic or hypoxic conditions for 21 days. Lungs, blood and bone marrow were collected. A) Illb, Il6, Ill8 and Tnfa expression was assessed by RT-qPCR. B) IL-6 protein expression was evaluated in whole lung and serum by ELISA. C) The numbers of blood monocytes and lymphocytes in hypoxic versus normoxic mice were determined by flow cytometry. D) The numbers of lung infiltrating neutrophils and interstitial macrophages in these mice were quantified by flow cytometry. $\mathrm{n}=5$ mice per condition. Data are shown as mean \pm s.e.m. ${ }^{*} \mathrm{P}<0.05$, $* * \mathrm{P}<0.01, * * * \mathrm{P}<0.005$, **** $\mathrm{P}<0.001$. 
Figure 3

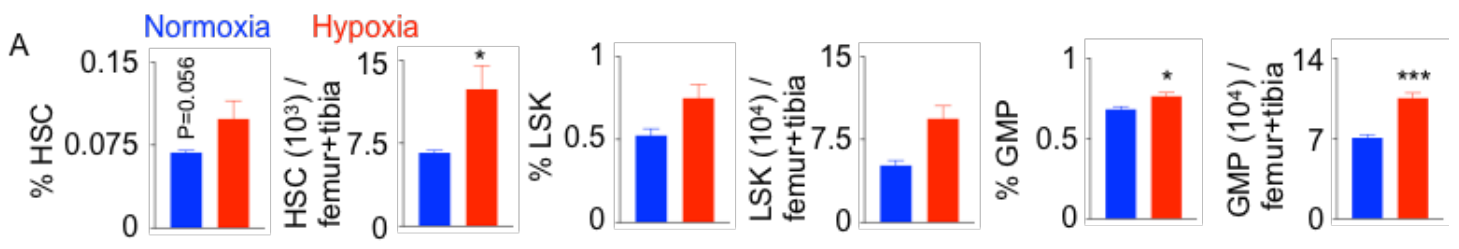

B

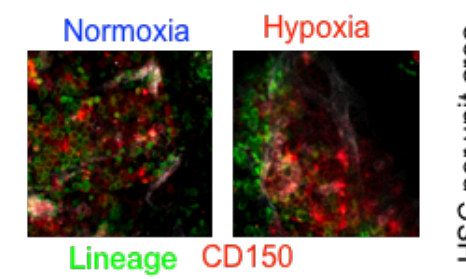

D LSK
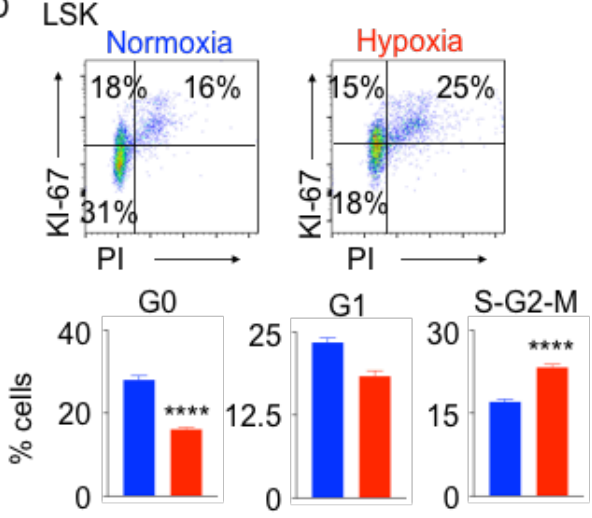

F Leukocytes Lymphos
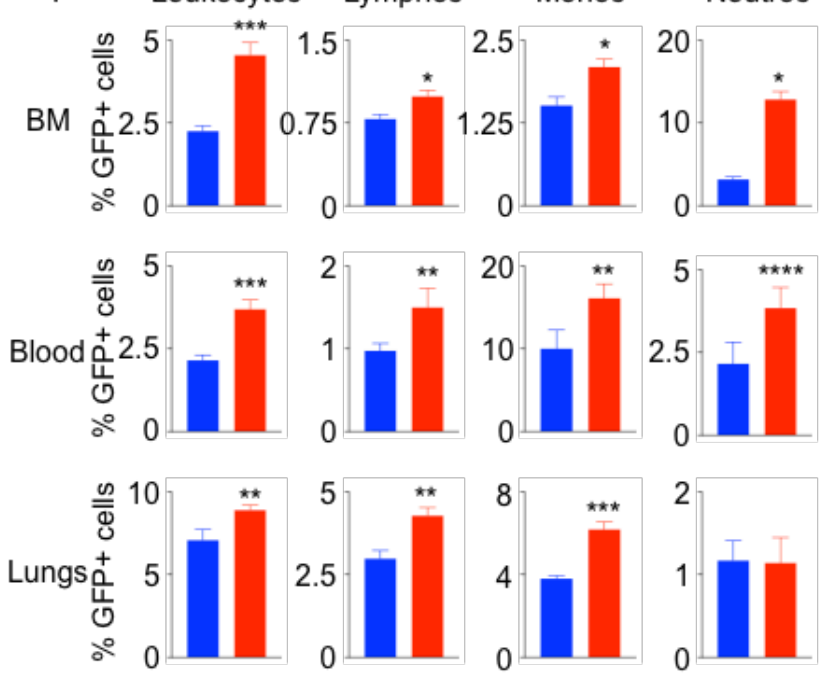
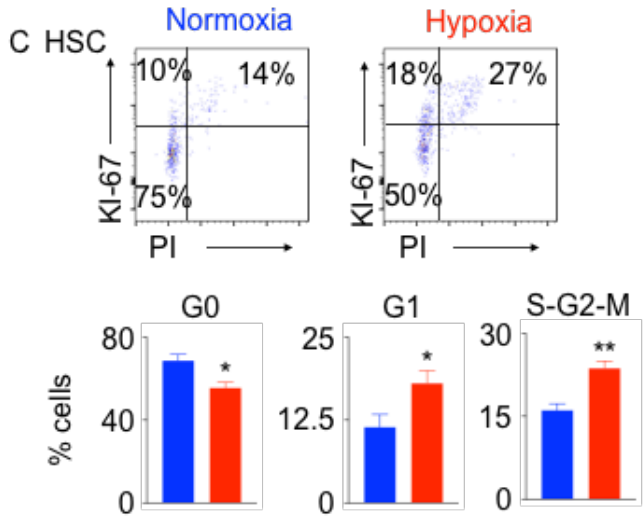

E GMP
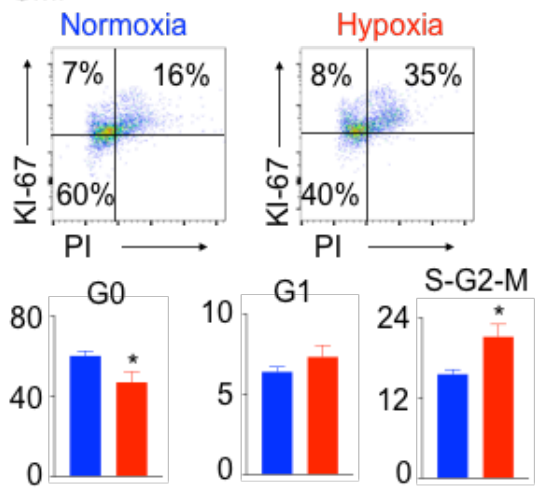

\section{Figure 3: Hypoxia drives HSPC into the cell cycle}

C57BL/6 mice were placed under either normoxic or hypoxic conditions for 21 days. Bone marrow was collected. The frequencies and numbers of the hematopoietic progenitors in the bone marrow were determined by flow cytometry (A) and whole mount confocal microscopy (B). The frequencies of HSC (C), LSK (D) and GMP (E) in the cell cycle stages were assessed using intracellular staining of Ki-67 and PI. (F) KikGR-GFP ${ }^{+}$ HSPC were adoptively transferred into mice housed in either normoxic or hypoxic conditions for seven days. $\mathrm{GFP}^{+}$progenies were quantified four days later. $\mathrm{n}=5$ mice per condition. Data are shown as mean \pm s.e.m. ${ }^{*} \mathrm{P}$ $<0.05, * * \mathrm{P}<0.01, * * * \mathrm{P}<0.005, * * * * \mathrm{P}<0.001$. 
Figure 4
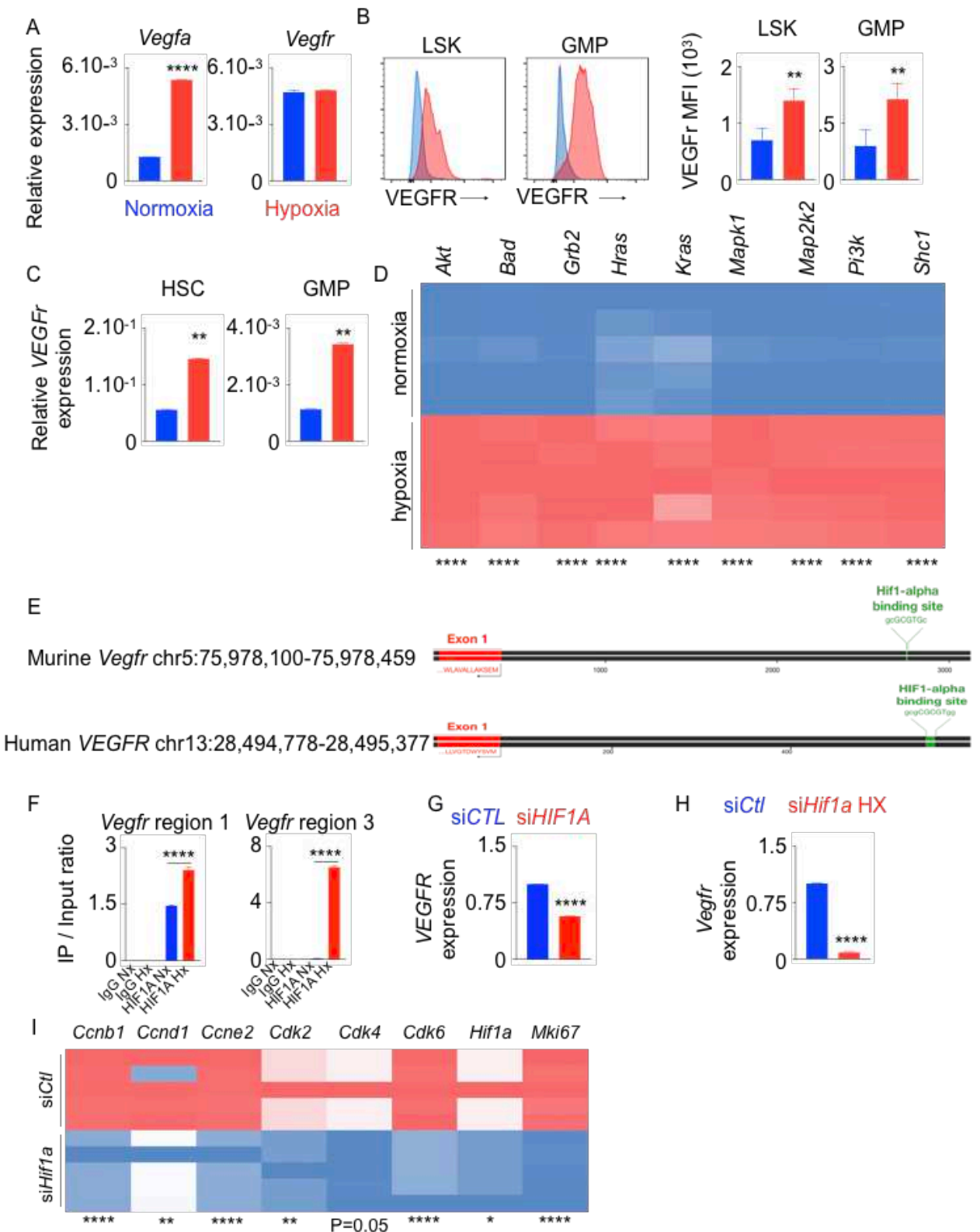

H siCtl siHif1a HX

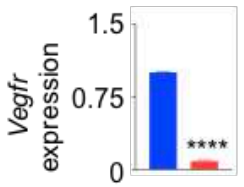

Figure 4: VEGFr expression is increased in HSPC in hypoxia

A-C) C57BL/6 mice were placed under either normoxic or hypoxic conditions for 21 days. Bone marrow was collected. A) Vegfa and Vegfr expression in the whole bone marrow was assessed by RT-qPCR. B) Vegfr expression was measured in bone marrow LSK and GMP by flow cytometry. The results are represented as mean fluorescent intensity (MFI). C) Vegfr gene expression was measured in sorted HSC and GMP by RT-qPCR. DF) HSPC were sorted from bone marrow of C57BL/6 mice and cultured in complete SFEM media in either normoxic or hypoxic conditions. D) Heatmap representing expression of genes downstream to Vegf/Vegfr in HSPC cultured under normoxic and hypoxic conditions. E) Schematic depicting HIF-1 $\alpha$ binding sites on both murine and human $V E G F r$ promoter regions. F) Confirmation of the binding of HIF-1 $\alpha$ to the murine $V e g f r$ promoter region assessed by ChIP qPCR. G-H) $V E G F r$ expression assessed in human (G) and murine (H) HSPC transfected with either siCTL or siHIF1A in hypoxic conditions. I) Heatmap showing the expression of the cell cycle genes in HSPC transfected with either siCtl or siHifla in hypoxic conditions. $\mathrm{n}=5$ mice per condition (A-C), 5 replicates per condition (D-I). Data are shown as mean \pm s.e.m. $* \mathrm{P}<0.05, * * \mathrm{P}<0.01, * * * * \mathrm{P}<0.001$. 
Figure 5

A Ccnb1 ${ }^{\star \star \star}$

Cond 1 ***

Cone1 ${ }^{\star \star \star}$

$C d k 2^{\text {*** }}$
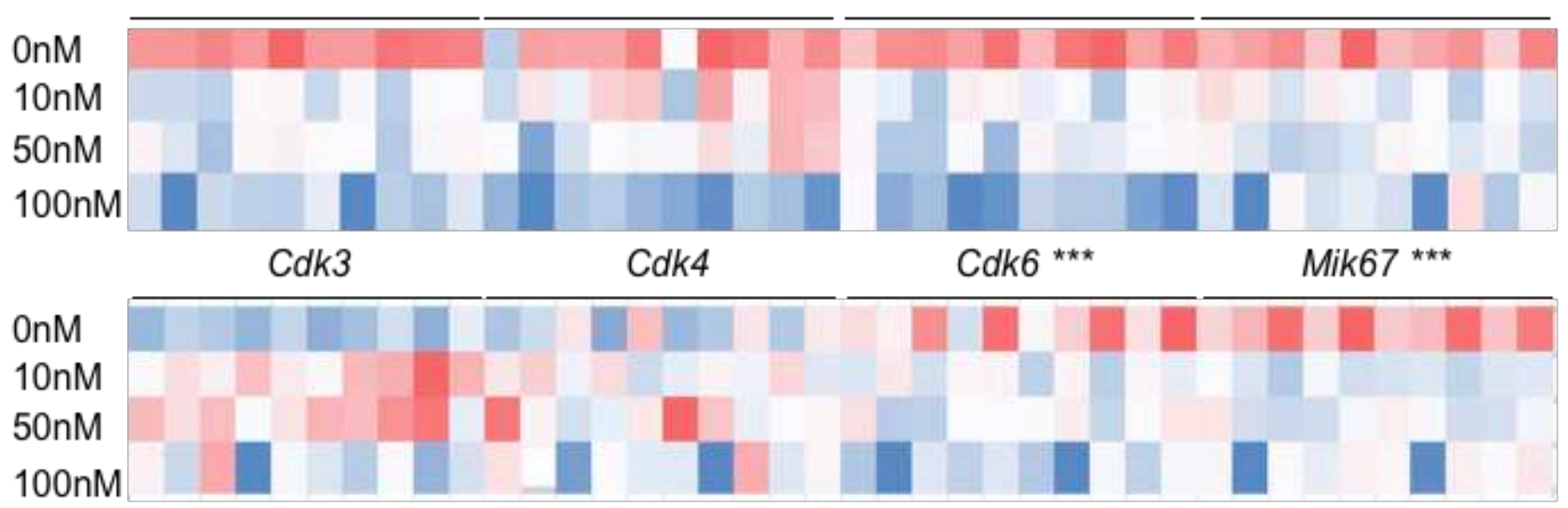

B

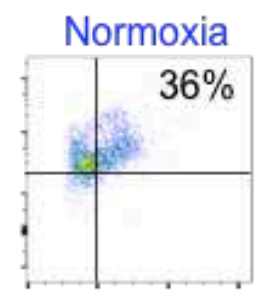

D Hypoxia

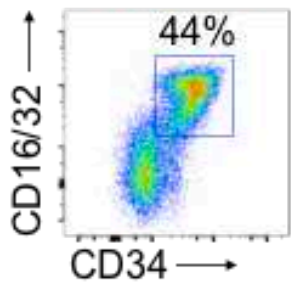

Hypoxia

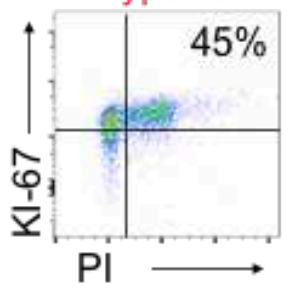

$\mathrm{PI} \longrightarrow$

Hypoxia

+VEGFr inhibitor

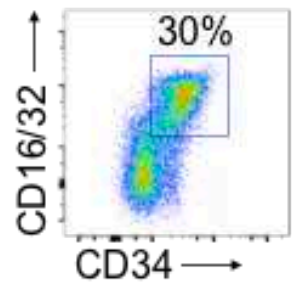

Hypoxia

+VEGFr inhibitor
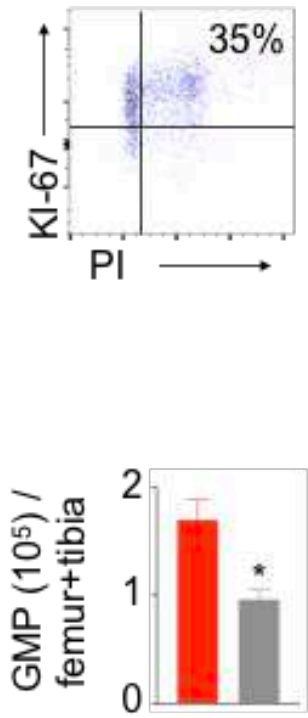

C

G0

G1

S-G2-M
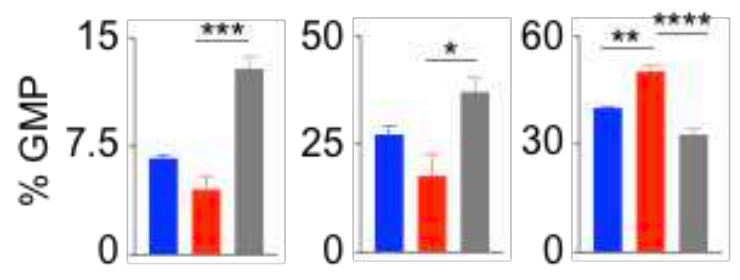

Figure 5: Vegfr inhibition decreased hematopoietic progenitor proliferation in hypoxia

A) Heatmap depicting expression of the cell cycle genes in mouse HSPC treated with increasing amounts of a Vegfr inhibitor (sugen, 10-100nM) or vehicle. B-D) C57BL/6 mice were treated with either sugen or vehicle diluent and placed in either normoxia or hypoxia for 21 days. Bone marrow cells were analyzed using flow cytometry to assess GMP proliferation $(B \& C)$ and number $(D) . n=5$ replicates or mice per condition. Data are shown as mean \pm s.e.m. $* \mathrm{P}<0.05, * * * \mathrm{P}<0.001, * * * * \mathrm{P}<0.001$. 


\section{Figure 6}

A Lymphoid lineage Myeloid lineage Total cells
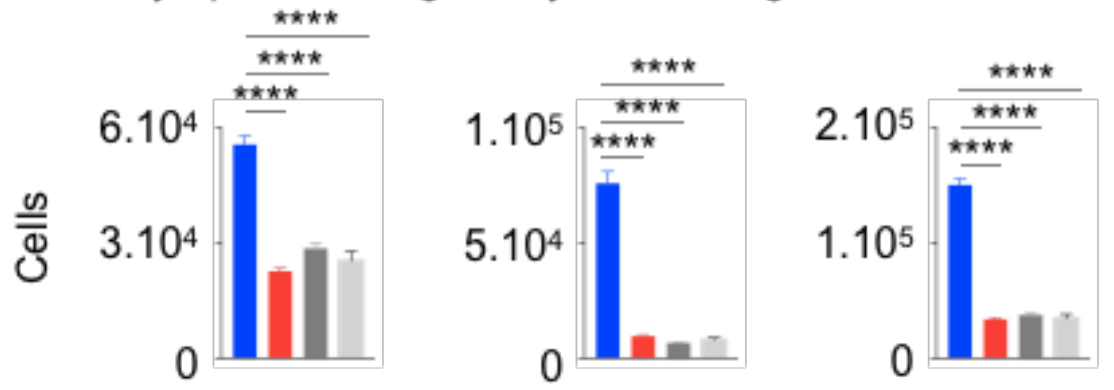

No treatment

10nM VEGFr inhibitor 50nM VEGFr inhibitor 100nM VEGFr inhibitor
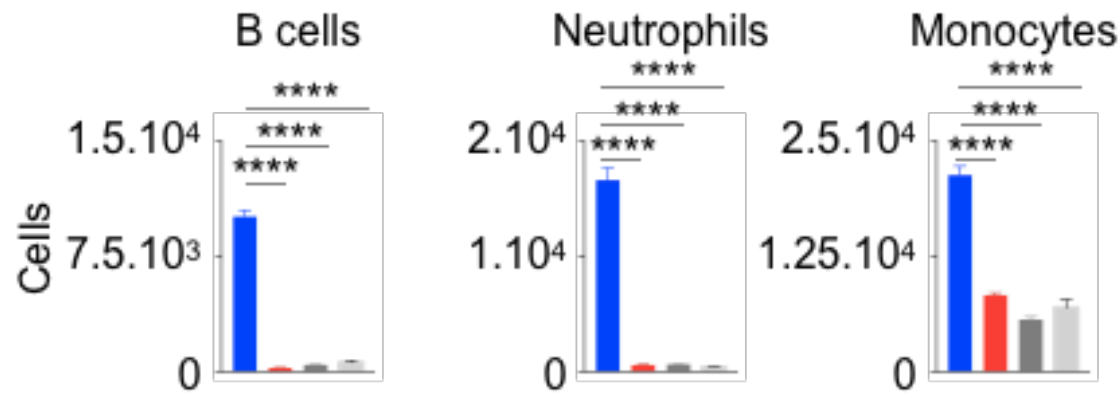

B Blood

Monocytes
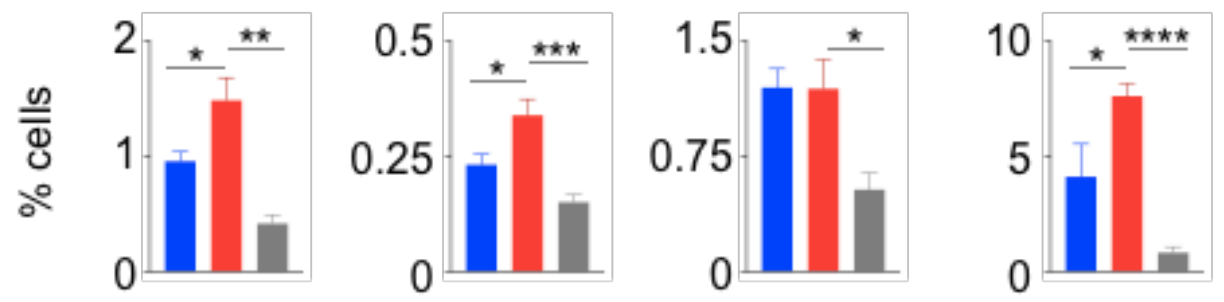

\section{Bone Marrow}

\section{Monocytes}

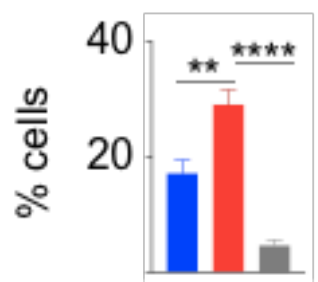

Neutrophils

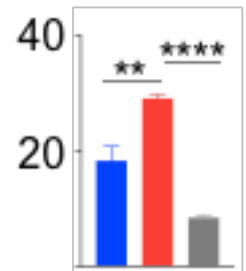

Normoxia

Hypoxia

Hypoxia

+VEGFr inhibitor

Figure 6: Vegfr inhibition decreased inflammation in hypoxia

A) Mouse HSPC were treated with increasing amounts of a Vegfr inhibitor (sugen, 10-100nM) or vehicle diluent (no treatment). Lineage commitment of HSPC was assessed by flow cytometry. B\&C) C57BL/6 mice were treated with either a Vegfr inhibitor (sugen) or vehicle diluent and placed in hypoxia for 21 days. Bone marrow and blood leukocytes were analyzed using flow cytometry. Percentages of blood (B) and bone marrow (C) monocytes and neutrophils were ascertained. $\mathrm{n}=5$ replicates or mice per condition. Data are shown as mean \pm s.e.m. $* \mathrm{P}<0.05, * * \mathrm{P}<0.01, * * * \mathrm{P}<0.001,{ }^{* * * *} \mathrm{P}<0.0001$. 



\section{Supplementary Files}

This is a list of supplementary files associated with this preprint. Click to download.

- 20211020Supplementalmaterial.pdf 